\title{
The "double dunk" technique for a right ventricle to pulmonary artery conduit implantation during the Norwood procedure reduces unintended shunt-related events
}

\author{
Tomasz Mroczek ${ }^{1}$, Julita Sacharczuk ${ }^{1}$, Rafał Żurek ${ }^{1}$, Aleksandra Morka ${ }^{1}$, Aleksandr Szypulski ${ }^{1}$, \\ Jerzy Jarosz' ${ }^{1}$ Maciej Śniechowski ${ }^{2}$, Janusz H. Skalski ${ }^{1}$ \\ 'Department of Paediatric Cardiac Surgery, Jagiellonian University Medical College, Krakow, Poland \\ ${ }^{2}$ Faculty of Physics and Applied Computer Science, AGH University of Science and Technology, Krakow, Poland
}

\begin{abstract}
Background: The introduction of a right ventricle to pulmonary artery conduit (RVPAc) during the Norwood procedure (NP) for hypoplastic left heart syndrome (HLHS) resulted in a higher survival rate, but also in an increased number of unintended pulmonary and shunt-related interventions.

Aim: To analyse how several modifications employed in RVPAc implantation during NP may influence the interstage course, unintended surgical or catheter-based interventions, and pulmonary artery development in a cohort of patients with HLHS.

Methods: We retrospectively analysed three groups of non-selected, consecutive neonates who underwent the NP between 2011 and 2014, with different RVPAc surgical techniques employed: Group I ( $=32)$ — left RVPAc with distal homograft cuff, Group II ( $n=28)$ — right RVPAc with distal homograft cuff, and Group III ( $n=41)$ — "double dunk," right reinforced $\operatorname{RVPAC}(\mathrm{n}=41)$.

Results: There were no intergroup differences in terms of age, weight, prevalence of aortic atresia, diameter of the ascending aorta, deep hypothermic circulatory arrest time, and hospital mortality rate (9.3\% vs. $14.2 \%$ vs. $7.3 \%$, respectively). There was a significant difference between the groups in the number of catheter-based interventions during the interstage period (34\% vs. $25 \%$ vs. $0 \%$, respectively, $\mathrm{p}<0.05)$ and/or concomitant surgical interventions $(17.2 \%$ vs. $4.1 \%$ vs. $2.6 \%$, respectively). The diameter of the pulmonary arteries was most homogenous in the third group.

Conclusions: The modified strategy of using the "double dunk," right reinforced RVPAc during the NP for HLHS significantly reduces the number of unintended catheter-based and surgical shunt-related reinterventions during the interstage period. This technique allows a more homogenous development of pulmonary arteries before the second, surgical stage.
\end{abstract}

Key words: hemi-Fontan procedure, hypoplastic left heart syndrome, Norwood operation

Kardiol Pol 2018; 76, 12: 1697-1704

\section{INTRODUCTION}

The renaissance of the concept of right ventricle to pulmonary artery conduit (RVPAC) as the source of pulmonary blood flow during the stage I Norwood procedure (NP), suggested originally by Norwood [1] and popularised by Japanese surgeons $[2,3]$, has positively affected the early [4], interim [5, 6], and long-term mortality rates $[7,8]$ in children with hypoplastic left heart syndrome (HLHS). Its potential haemodynamic advantages have been also partially explained and confirmed by computer simulations [9]. However, initial experience revealed an increased rate of unintended RVPA shunt-related interventions. In our experience, similar observations and a better understanding of their nature have led to several modifications aimed at eliminating this phenomenon. The objective of this study is to present our institutional experience demonstrating the way in which the changes in the technique

\section{Address for correspondence:}

Tomasz Mroczek, MD, PhD, Department of Paediatric Cardiac Surgery, Jagiellonian University Medical College, ul. Wielicka 265, 30-663 Kraków, Poland, tel: + 481265810 23, fax: +48 1265739 47, e-mail: t_mroczek@hotmail.com 
Table 1. Patients' characteristics at stage I Norwood operation

\begin{tabular}{lcccc|} 
Parameter & $\begin{array}{c}\text { Left RVPAc } \\
(\mathbf{n}=\mathbf{3 2})\end{array}$ & $\begin{array}{c}\text { Right RVPAc } \\
(\mathbf{n}=\mathbf{2 8})\end{array}$ & $\begin{array}{c}\text { Right reinforced } \\
\text { RVPAc }(\mathbf{n}=\mathbf{4 1})\end{array}$ & $\mathbf{p}$ \\
\hline Age [days] & $10.7 \pm 7.22$ & $12.1 \pm 6.94$ & $9.8 \pm 7.23$ & NS \\
Weight [kg] & $3.3 \pm 0.27$ & $3.3 \pm 0.63$ & $3.2 \pm 0.35$ & NS \\
Gestational age [weeks] & $38.3 \pm 1.47$ & $39.9 \pm 1.42$ & $38.9 \pm 1.23$ & NS \\
Prenatal diagnosis & 53.1 & 57.4 & 58.5 & NS \\
Aortic atresia & 50 & 57.1 & 3.7 & NS \\
Diameter of ascending aorta [mm] & $3.5 \pm 1.72$ & $3.1 \pm 2.31$ & $4.4 \pm 1.52$ & NS \\
Diameter of aortic arch [mm] & $4.6 \pm 1.11$ & $4.8 \pm 1.32$ & NS \\
\hline
\end{tabular}

Data are presented as percentage or mean \pm standard deviation. RVPAc — right ventricle to pulmonary artery conduit; NS — non-significant

of RVPAC implantation may affect the results and reduce the necessity of unintended catheter-based and surgical reinterventions after the NP.

\section{METHODS}

The Hospital Institutional Review Board approved the study, and due to its retrospective design the obligation to obtain informed consent was waived. An inception cohort of 101 consecutive neonates with HLHS, diagnosed and operated on in the Department of Paediatric Cardiac Surgery, Jagiellonian University Medical College, Krakow, Poland, between 2011 and 2014, were enrolled. The available parameters from patients' records were analysed (Table 1 ).

\section{Surgical technique}

RVPAc implantation technique

The total cohort of examined patients was divided into three groups according to the technique of RVPAc implantation used during the NP. All RVPAcs were $5 \mathrm{~mm}$ in diameter, except for three cases in Group III, in which 6-mm shunts were employed when the patient's body weight was above $3.5 \mathrm{~kg}$. The implantation of the proximal aspect of the RVPAc was done after choosing the weak area of the major coronary in the right ventricular (RV) outflow tract; however, some distance had to be maintained to avoid damaging the pulmonary artery valve.

In Group I, after the placement of a retraction suture, ventriculotomy was made initially with a blade and subsequently with a 5-mm punch. After our initial experience with severe proximal stenosis, a more extended ventriculotomy was employed (ventriculotomy had the shape of a truncated cone, with a wider diameter in the inner layer of the RV free wall). A 5-mm polytetrafluoroethylene tube was sutured perpendicularly to the surface of the RV; the graft was located to the left of the ascending aorta, and the distal part of the RVPAc was sutured to the pulmonary confluence after previous augmentation with a homograft cuff, prepared shortly before the operation. Usually, distal anastomosis was done during the cooling phase. The length of the shunt was approximately $35 \mathrm{~mm}$.
In Group II, the technique of implantation of the proximal and distal aspects of the RVPAc was identical as in Group I; however, the graft was located to the right of the ascending aorta. The decision regarding the side on which the shunt should be located with respect to the ascending "neoaorta" was at the discretion of the surgeons, and there were a few patients from Groups I and II in whom the procedure was performed at the same stage of the project. In some cases the anatomical configurations and the relationship between the reconstructed ascending aorta and the pulmonary confluence were thought to be unsuitable for the right-sided shunt location. The mean length of the right-sided RVPAc was approximately $55 \mathrm{~mm}$ (Fig. 1).

In Group III, commercially available 5-mm shunts reinforced with rings were employed and were located to the right of the ascending aorta. This group consisted of 41 consecutive patients operated on most recently. During the rewarming phase, a small cylindrical-shaped ventriculotomy was created with a $4.5-\mathrm{mm}$ punch (smaller than in the previous groups). The proximal aspects of the conduit were inserted into the free wall of the RV. The polytetrafluorethylene membrane was cut sharply at the level of the first ring. For proximal fixation of the conduit, three 5-0 polypropylene sutures were introduced through the full thickness of the RV (including the endocardium) and fixed only to the third ring of the shunt without the perforation of the shunt membrane. Consequently, part of the shunt was dunked into the RV at a distance equal to the thickness of the RV. After the sutures were tightened, one of them was used as a superficial, haemostatic suture tied exclusively to the third ring, located at the level of the epicardium. The distal aspect of the shunt was sutured directly to the anterior wall of the pulmonary confluence, following previous direct suturing of the pulmonary trunk opening. When the RVPAc was anastomosed directly to the pulmonary arteries, an approximately 1.5-mm-long shunt membrane margin was left behind the last ring as a protruding, mechanical barrier, working as a stent and preventing tissue growth into the conduit (distal "dunk" technique). In neonates with low birth weight and a small 


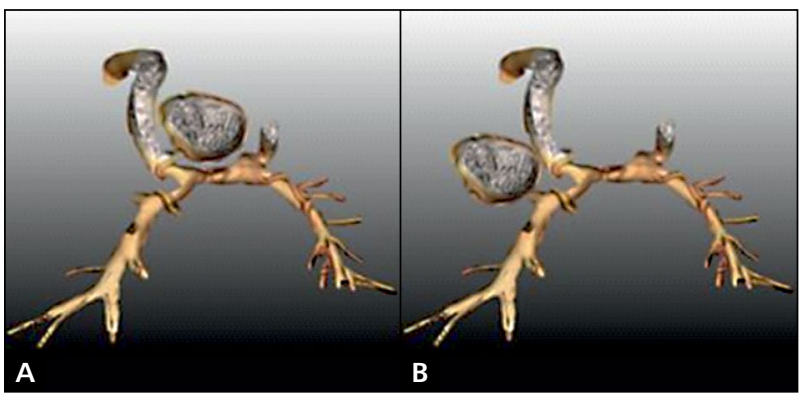

Figure 1. A. Computed tomography-angiogram showing the relationship between the ascending aorta and pulmonary arteries after the modified Norwood procedure, when the right ventricle to pulmonary artery conduit is located to the right with respect to the ascending aorta; $\mathbf{B}$. Simulation suggesting that the right pulmonary artery extending to the right, but also posteriorly, is less vulnerable to potential compression of the ascending aorta, and the proximal aspect of the left pulmonary artery is more amenable to reconstruction during the second stage

diameter of the pulmonary confluence, the distal shunt was augmented with a homograft patch and implanted into the anterior aspect of the native pulmonary confluence. In all patients with a right-sided location of the RVPAc (Groups II and III), a free whetstone-like patch obtained from the polytetrafluorethylene vascular shunt was left in the midline, covering the free wall of the RV and the shunt, crossing at some point the midline of the sternum (Fig. 2-4).

\section{Norwood procedure}

The NP was performed using deep hypothermia and circulatory arrest. The technique of aortic reconstruction was constant throughout the entire study. Our preference was to reconstruct the aorta with a pulmonary homograft patch. Routine septectomy was performed through the atrial cannulation site. The right atrium was not opened except in eight cases in which various forms of tricuspid valve repair were performed, two patients with anomalous pulmonary vein connection, and two patients with intact interatrial septum. The haematocrit level was maintained at around $30 \%$. As soon as the postoperative bleeding had ceased, a continuous heparin infusion at a dose of $10 \mathrm{U} / \mathrm{kg} / \mathrm{h}$ was initiated. It was then increased to $25 \mathrm{U} / \mathrm{kg} / \mathrm{h}$ within a few hours, and maintained for as long as the central venous line was in place. When enteral feeding became possible (usually on the first postoperative day), low-dose acetylsalicylic acid (2 mg/kg/day) was started.

The second-stage operation

The hemi-Fontan procedure was the method of choice during the second stage of the surgical treatment. The children were not routinely subjected to catheterisation before the second stage, except for situations when some interventions

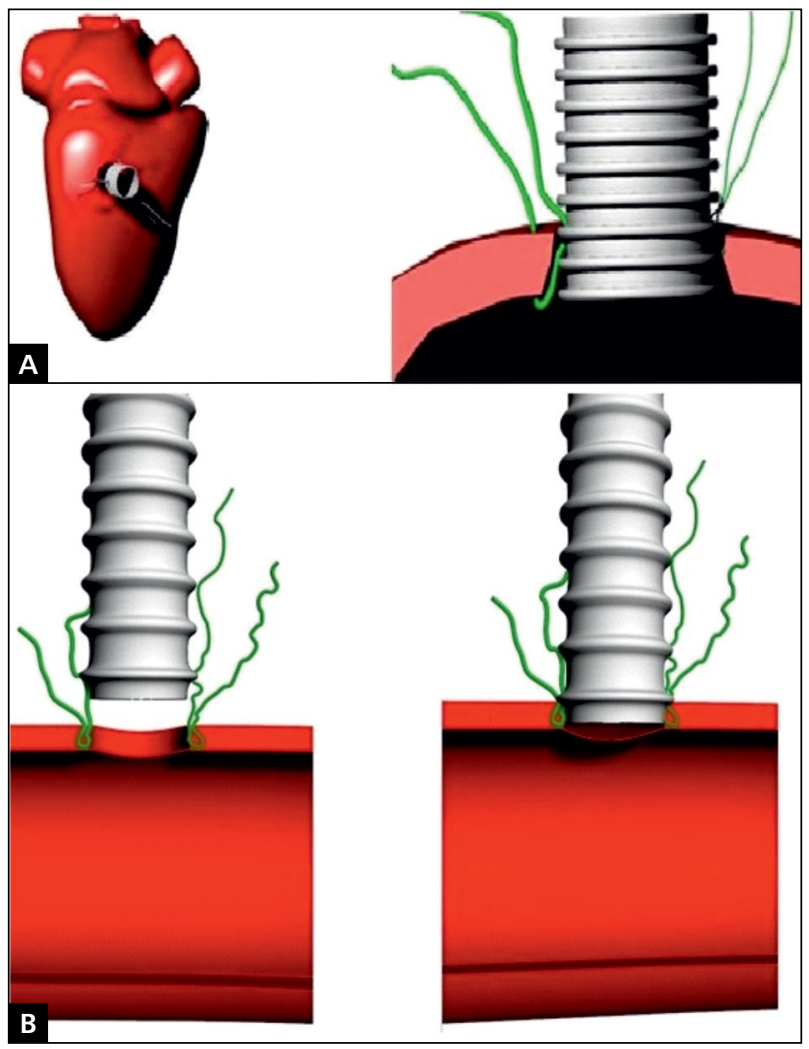

Figure 2. A. Method of proximal fixation of the right ventricle to pulmonary artery conduit (RVPAc) into the free wall of the right ventricle during the Norwood procedure; B. Technique of "distal dunk" implantation of RVPAc into the pulmonary artery. The sutures were placed in the ring and pulmonary wall, while the membrane is protruding into the lumen of the pulmonary artery.

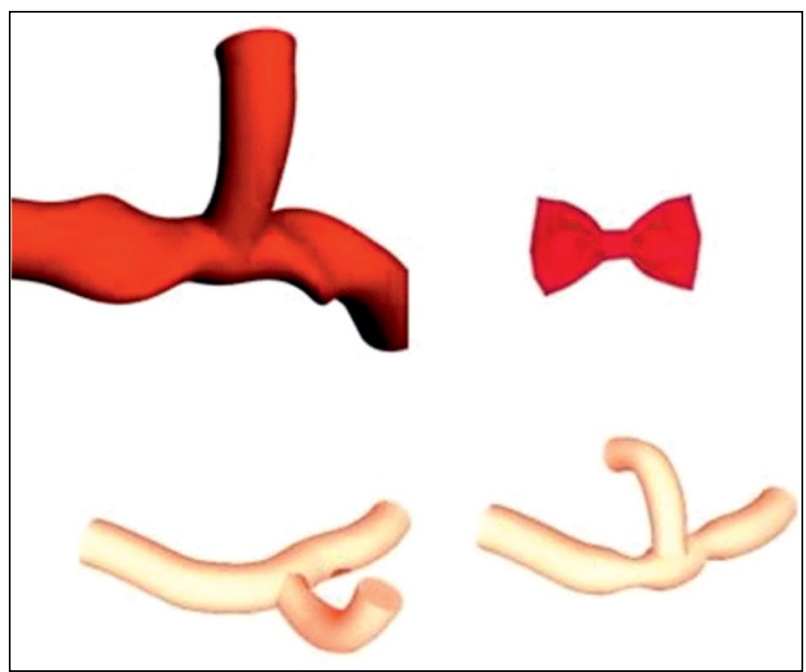

Figure 3. The potential mechanism of the so-called "bow-tie" effect. Twisting of the proximal aspect of the pulmonary arteries caused by the right ventricle to pulmonary artery conduit reaching the pulmonary confluence from the anterior aspect, when anastomosed to the opening of the pulmonary trunk 


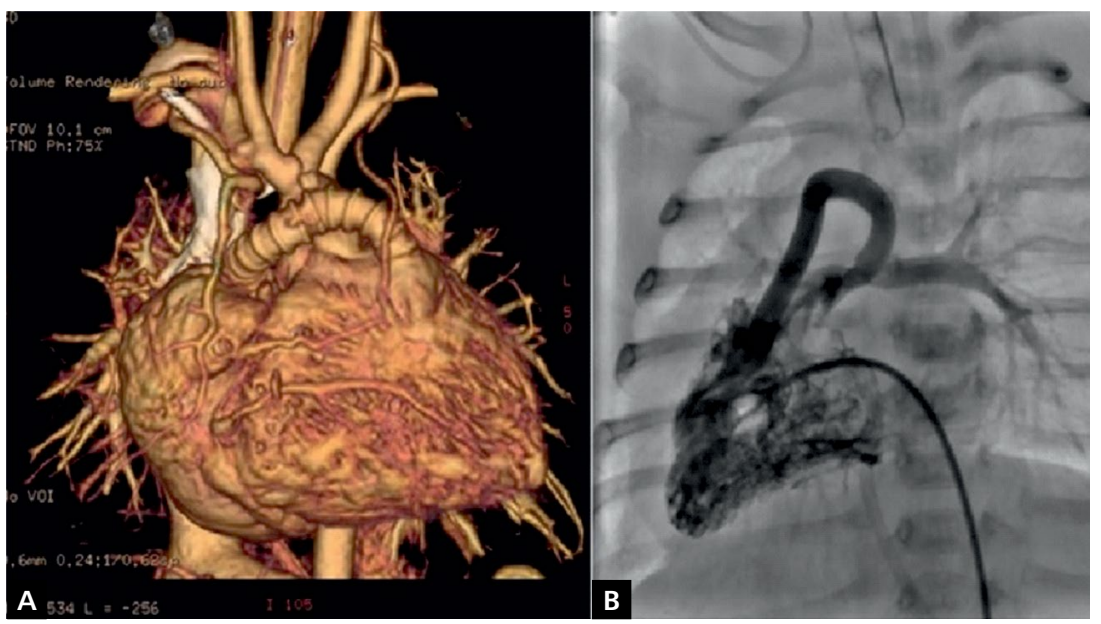

Figure 4. A. Computed tomography-angiogram demonstrating the right reinforced right ventricle to pulmonary artery conduit (RVPAC) in a child with hypoplastic left heart syndrome (HLHS) after the modified Norwood procedure (NP). The RVPAc reaches the pulmonary confluence from the anterior direction, rather than from the inferior, as in the anatomic position of the pulmonary trunk before the NP; B. Angiogram of a HLHS patient with "situs inversus" after the modified NP with the reinforced "right" (on the left side) RVPAc. The diameter of the entire RVPAc is constant along the conduit

Table 2. Patients' characteristics at stage II hemi-Fontan palliation

\begin{tabular}{lcccc} 
Parameter & $\begin{array}{c}\text { Left RVPAC } \\
\text { (Group I; } \mathbf{n}=\mathbf{2 5} \text { ) }\end{array}$ & $\begin{array}{c}\text { Right RVPAC } \\
\text { (Group II; } \mathbf{n}=\mathbf{2 1} \text { ) }\end{array}$ & $\begin{array}{c}\text { Right reinforced RVPAc } \\
\text { (Group III; } \mathbf{n}=\mathbf{3 8} \text { ) }\end{array}$ & $\mathbf{p}$ \\
\hline Age [months] & $6.4 \pm 1.7$ & $6.2 \pm 1.9$ & $5.9 \pm 2.0$ & NS \\
Weight [kg] & $6.3 \pm 0.99$ & $6.2 \pm 0.82$ & $6.2 \pm 0.89$ & NS \\
Systemic sat. [\%] & 72.2 & 73.7 & 75.1 & NS \\
RV dysfunction & 3 & 2 & 1 & NS \\
Additional procedures & 2 & 4 & 5 & NS \\
Mortality rate & $1 / 25$ & $1 / 21$ & 0 & NS \\
CPB time [min] & $106 \pm 32$ & $98 \pm 41$ & $94 \pm 37$ & $<0.05$ \\
Mean DHCA [min] & $42 \pm 9$ & $33,37,31^{*}$ & 0 & Waived \\
\hline
\end{tabular}

Data are presented as number, percentage or mean \pm standard deviation. *Only three patients had periods of circulatory arrest; CPB — cardio- pulmonary bypass; DHCA — deep hypothermic circulatory arrest; RV — right ventricle; RVPAc — right ventricle to pulmonary artery conduit; sat. - oxygen saturation

needed to be performed or echocardiographic assessment was not diagnostic.

The diameters of the pulmonary arteries included in the analysis were assessed by echocardiography; the measurements included the proximal aspect (at the site of the anastomosis with the conduit) and the distal segment, immediately before pulmonary artery bifurcation. The accepted values represent mean values of three measurements performed by the same cardiologist (A.M.) (Table 2).

\section{Statistical analysis}

The data were analysed using the statistical software package Statistica 10 (Statsoft Inc., Tulsa, OK, USA). Standard descriptive statistics were used for the characterisation of the groups. Continuous variables were expressed as means (standard deviations), and comparative analyses were performed with the use of the Tukey multiple comparisons of means or the Wilcoxon signed-rank test. Nelson-Aalen analysis of cumulative hazard function of reinterventions was performed. The probability value of less than 0.05 was taken to represent a statistically significant difference between the groups.

\section{RESULTS}

We present a cohort of patients with HLHS divided into three groups according to the strategy of RVPAc implantation. There were no significant differences between the groups in terms of age, weight, diameter of ascending aorta, prevalence of aortic atresia, and concomitant diagnosis at the time of the NP. 


\section{Shunt-related catheter-based and surgical interventions}

During the interstage period, some patients underwent catheter-based interventional and surgical procedures.

In Group I ( $\mathrm{n}=32), 10$ patients underwent interventional catheterisations. The interventions were mostly related to the proximal aspects of the RVPAc, except for one case, in which balloon angioplasty of the distal shunt and proximal aspects of the pulmonary arteries was performed. In two cases, ballooning of the proximal aspect was effective, whereas in three cases it had to be followed by stent implantation into the proximal aspect of the RVPAc. In four cases, balloon angioplasty of the proximal aspect of the RVPAc was ineffective, and the patients were referred for surgical shunt exchange. Surgical interventions consisted of RVPAc exchange with concomitant pulmonary artery angioplasty. A 5-mm RVPAc was used for the exchange in all patients except for one (weighing $4.9 \mathrm{~kg}$ ), in whom a 6-mm shunt was applied.

In Group II ( $\mathrm{n}=28)$ there were six catheter-based interventions and one surgical intervention. The proximal part and the midportion of the RVPAc were stented in two cases each, and in two other cases angioplasty involving only the proximal aspects of the pulmonary arteries was employed. In one patient at the age of 2.1 months, the proximal parts of the pulmonary arteries were surgically reconstructed due to persistent hypoxia.

In Group III ( $\mathrm{n}=41)$ no patients required pulmonary artery interventions. One patient demonstrated significant tricuspid regurgitation and hypoxaemia. When the baby was two months old and weighed $3.9 \mathrm{~kg}$ it underwent an interstage intervention consisting of partial annuloplasty and the RVPAC exchange from a 5-mm to a 6-mm shunt.

Overall, the number of catheter-based interventions during the interstage period was significantly lower in Group III as compared to the other two groups ( $34 \%$ vs. $25 \%$ vs. $0 \%$, respectively for Groups I, II, and III, p < 0.05) as was the number of concomitant surgical interventions $(17.2 \%$ vs. $4.1 \%$ vs. $2.6 \%$, respectively; Fig. 5).

\section{Non-shunt-related interventions}

In Group I, four patients underwent balloon aortoplasty, and in two other a stent was implanted into the aortic isthmus. In Group II, there were two cases of balloon aortoplasty and two stent implantations were performed.

In Group III, one patient underwent an independent interventional procedure of aortoplasty, whereas in three other, in whom the stenosis was borderline, balloon angioplasty was performed through the aortic cannula during the hemi-Fontan procedure, in a hybrid room, after completion of the surgical procedures.

\section{Survival rate}

The 30-day mortality rates were $9.3 \%$ in Group I (3/32), $14.2 \%$ in Group II (4/28), and $7.3 \%$ in Group III. The causes
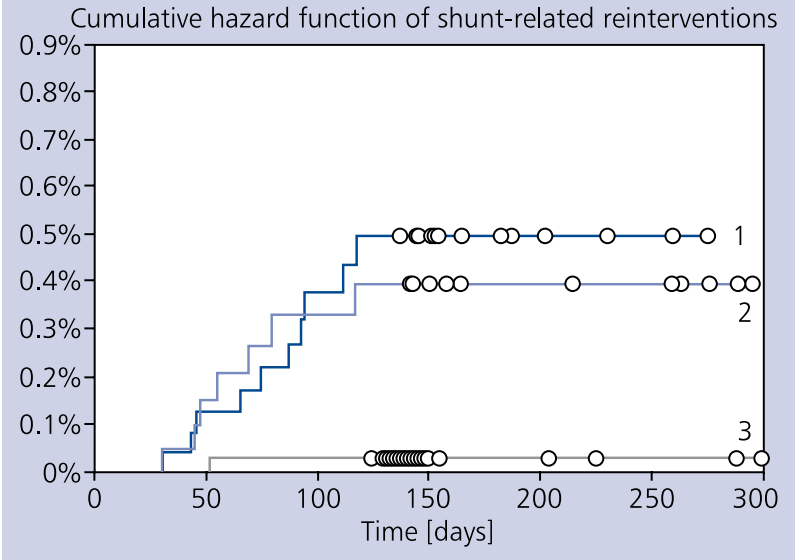

Figure 5. Nelson-Aalen analysis of cumulative hazard ratio of right ventricle to pulmonary artery conduit (RVPAC)-related reintervention between the Norwood operation and second-stage procedures in patients with hypoplastic left heart syndrome. 1 - Group I (left RVPAc); 2 - Group II (right RVPAc); 3 - Group III (right reinforced modified RVPAc); $p=0.001$ between Groups I, II and III

of death in Group I were as follows: ventricular dysfunction (one patient), sudden RVPA shunt occlusion (one patient), and sepsis and multi-organ dysfunction (one patient). In Group II there were four deaths; one patient presented with severe RV dysfunction and while on extracorporeal membrane oxygenation (ECMO), angiography revealed a huge left coronary artery to left ventricle fistula, one patient died suddenly, probably due to shunt occlusion, and one patient with dysplasia and insufficiency of the tricuspid and pulmonary valves demonstrated low cardiac output and eventually multi-organ failure. One patient developed shunt occlusion and died in spite of an emergency reoperation. In Group III there were three deaths; one involved a patient with intact interatrial septum, one patient with congenital pneumonia was operated at 45 days of age and developed sepsis, and one patient died due to ventricular dysfunction that did not resolve in spite of ECMO.

\section{Concomitant procedures}

Several types of concomitant procedures were performed during the stage I Norwood. In Group I, two patients underwent commissuroplasty of the tricuspid valve, and one patient underwent aortic valve repair due to significant insufficiency as a consequence of balloon valvuloplasty performed in another institution before the NP. In Group II, repair of total supracardiac anomalous pulmonary venous connection was performed at the time of the NP (commissuroplasty of the tricuspid valve in two patients, and partial annuloplasty in two patients). One patient with the right aortic arch needed a modified NP, and one patient had situs inversus, and so had 
Table 3. Diameters of pulmonary arteries assessed before the hemi-Fontan operation

\begin{tabular}{|lcccc|}
\hline Parameter & Group I & Group II & Group III & p \\
\hline Proximal LPA [mm] & $3.91 \pm 0.2$ & $4.01 \pm 0.03$ & $4.21 \pm 0.2$ & $<0.05$ between I and III group \\
Distal LPA [mm] & $4.89 \pm 0.3$ & $4.97 \pm 0.2$ & $5.08 \pm 0.3$ & $<0.05$ between I and III group \\
Proximal RPA [mm] & $3.97 \pm 0.2$ & $4.04 \pm 0.3$ & $4.33 \pm 0.2$ & NS \\
Distal RPA [mm] & $5.48 \pm 0.1$ & $5.45 \pm 0.2$ & $5.65 \pm 0.3$ & NS \\
\hline
\end{tabular}

Data are presented as mean \pm standard deviation. LPA — left pulmonary artery; RPA — right pulmonary artery

to undergo a mirror-image NP. In Group III, one patient had situs inversus, three patients underwent partial annuloplasty of the tricuspid valve, and in three other patients commissuroplasty was performed. The chest was left-opened or reopened within a short time after the NP in $25 \%, 21.4 \%$, and $14.6 \%$ of patients in Groups I, II, and III, respectively ( $p>0.05$ ).

\section{Hemi-Fontan procedure}

Hemi-Fontan procedure was the method of choice during the second stage of the Fontan pathway in our institution.

In Group I, there was one interim death (unknown reason) and three patients had the stage II procedures performed at another centre. In Group II, there was one interim death (shunt-related), and two patients had the stage II operations performed elsewhere. There was no interim mortality in Group III.

In total, 25 patients in Group I underwent the hemi-Fontan procedure and four of them, in whom left superior vena cava was present, also underwent the bidirectional Glenn procedure on the left side. In Groups II and III, the hemi-Fontan procedure was performed in 21 and 38 patients, respectively, with two patients also undergoing the bidirectional left-Glenn in each group. Two patients (one from Group I and one from Group II) underwent a rescue hemi-Fontan at the age of 4.5 and 4.8 months, respectively, because of rapidly progressing cyanosis. In both cases, the interatrial communication was enlarged; there were no unplanned operations in Group III (Table 3).

\section{DISCUSSION}

Our study demonstrates that refinements in the surgical technique of RVPAc implantation may significantly reduce the need for catheter-based and surgical shunt-related reinterventions shortly after the NP and during the interim period. The new concepts of the RVPAc implantation allow better development of the pulmonary arteries and make the surgical technique more reproducible and straightforward. This is very promising in terms of increasing evidence indicating that the RVPAc has a favourable effect on long-term survival and probably no deleterious effect on the RV function and incidence of tricuspid valve insufficiency in children with HLHS after the NP. The modifications to be considered include the technique of proximal and distal anastomosis, as well as the location and reinforcement of the shunt. The main conclusion from the Single Ventricle Reconstruction trial and three-year re-evaluation of this multi-centre, randomised cohort of patients was a higher incidence of unintended shunt-related interventions between the first and second stages of surgical palliation [10]. In our study, we were able to show the kinds of modifications of the RVPAc implantation that may lead to a significant reduction of unintended interventions before the first and second stages [11].

\section{Proximal aspect of the RVPAc}

The majority of shunt-related reinterventions, especially in Group I, occurred due to stenosis of the proximal aspect of the RVPAc. The mechanisms responsible for stenosis are multifactorial: neointima formation, scar tissue migration into the shunt, kinking of the conduit, and thrombus formation, limiting the blood flow. Some patients developed subshunt stenosis, which was not seen during the operation but was significant when the turgor and contractility of the RV reached the appropriate value. Additionally, when the RVPAc was located to the right with respect to the ascending aorta (Group II), some degree of stenosis of the midportion of the shunt was also observed. To prevent it, we resorted to the implantation of the RVPAc reinforced with rings [12]. After some initial experience, it was clear that this type of prosthesis may be extremely helpful in managing the proximal anastomotic stenosis. Independently of Tweddell et al. [13] and Hasaniya et al. [14], we developed our own technique of "dunking" and proximal fixation of the RVPAc with three single sutures through full thickness of the RV wall, including the endocardium, and the additional superficial purse-string haemostatic suture. The role of three single sutures is not only to fix the shunt, but also to keep the injured myocardium away from the lumen of the RVPAc, which — we believe - may prevent scar tissue migration into the lumen of the shunt. That kind of proximal RVPAc fixation resulted in a significantly smaller, cylindrical (instead of cone-shaped) ventriculotomy [15]. In our study, the "dunk" part of the conduit was left in place during the removal of the remainder of the RVPAc. Baird et al. [16] suggest that complete removal of the conduit during the second stage palliation may be helpful in preserving the regional RV function. This suggestion needs to be analysed in the future. 


\section{Switch from the left to right RVPAc}

The significant modification of the RVPAc implantation technique was the transfer of the implantation site from the left to the right side of the ascending aorta. The main disadvantage of the left-sided location of the RVPAc is the difficulty in reaching the pulmonary confluence, the time taken to do so, and the risk of left phrenic nerve injury and bleeding during the stage II operation. Relocation of the shunt from the left to the right side of the "neoaorta" resulted in a much easier access to the pulmonary arteries during the second stage, especially when the proximal aspects of the pulmonary arteries were hypoplastic, requiring augmentation of the proximal aspect of both the left and right pulmonary arteries. What seemed important to us when the right RVPAc option was chosen was the fact that the true native confluence was relocated to the right. The natural course of the left pulmonary artery is to the left and posteriorly, which makes the segments of the pulmonary artery situated behind the ascending aorta less vulnerable to compression, or at least more compliant to preparation and reconstruction. Thus, the potential stenotic segments of the pulmonary arteries are relocated to the right and are easier to reconstruct during the second stage, if necessary.

\section{Distal aspect of the RVPAC}

The crucial issue regarding distal RVPAC anastomosis is the stenosis of the proximal aspects of the pulmonary arteries ("bow-tie" effect), which, in observations and computer simulations, is the result of twisting of the proximal aspects of both pulmonary arteries. The native pulmonary trunk is directed downward, whereas during the NP, suturing the RVPAc to the opening the pulmonary trunk results in a 90-degree twist in the anterior direction because of constraint from the RVPAc polytetrafluorethylene tube. To eliminate the effect of torsion, we started to employ primary closure of the distal opening of the pulmonary trunk, and the polytetrafluoroethylene tube was sutured directly to a 5-mm hole created with a punch in the anterior aspect of the previous pulmonary trunk. We believe that this modification is crucial in preventing the twisting of the proximal pulmonary arteries. Torsion of the proximal aspect of the pulmonary arteries directly reduces the diameter of the vessels, but also interferes with the local shear stress, influencing the growth potential. Another crucial point is "dunking" the distal aspect of RVPAc into the pulmonary artery to prevent any tissue migration into the lumen of the shunt. Reinforced shunt also may stent the place of implantation. This modification was previously described by Mascio and Spray [17]. Choosing the hemi-Fontan procedure as the second stage allows a routine augmentation of proximal aspects of pulmonary arteries.

We believe that the changes in the outcomes resulting from the implementation of the new RVPAc implantation technique are so significant that the cohorts of patients currently under study differ enough from previously studied groups to mandate the update or renewal of pending projects [18]. This will elucidate not only the development of the pulmonary arteries, but also the long-term consequences of the diminished $\mathrm{RV}$ ventriculotomy and better haemodynamic performance $[19,20]$. So far, a higher rate of reinterventions is the main known disadvantage of the RVPAc vs. the Blalock-Taussig shunt used in conjunction with the NP. Neutralisation of this effect through technical refinements may be a sufficiently strong argument influencing the choice of the pulmonary blood flow pattern used during the NP. Also, the development of pulmonary arteries needs to be re-evaluated in most modern cohorts of patients who underwent the NP with RVPAc.

In conclusion, the modified strategy of using the "double dunk" right reinforced RVPAc during the NP for HLHS significantly reduces the number of catheter-based and unintended surgical shunt-related reinterventions during the interstage period. This strategy allows a more homogenous development of pulmonary arteries before the second, surgical stage. Further analyses are necessary for the assessment of this strategy in terms of long-term follow-up after Fontan completion in patients with HLHS.

\section{Acknowledgements}

This project was funded in part by a grant (no. K/ZDS/004536) from the Jagiellonian University, Krakow, Poland (to T.M., J.S.).

\section{Conflict of interest: none declared}

\section{References}

1. Norwood WI, Lang P, Casteneda AR, et al. Experience with operations for hypoplastic left heart syndrome. J Thorac Cardiovasc Surg. 1981; 82(4): 511-519, indexed in Pubmed: 6168869.

2. Imoto Y, Kado H, Shiokawa Y, et al. Experience with the Norwood procedure without circulatory arrest. J Thorac Cardiovasc Surg. 2001; 122(5): 879-882, doi: 10.1067/mtc.2001.116948, indexed in Pubmed: 11689791.

3. Sano S, Ishino K, Kawada M, et al. Right ventricle-pulmonary artery shunt in first-stage palliation of hypoplastic left heart syndrome. J Thorac Cardiovasc Surg. 2003; 126(2): 504-509, doi: 10.1016/s0022-5223(02)73575-7.

4. Malec E, Januszewska K, Kolcz J, et al. Right ventricle-to-pulmonary artery shunt versus modified Blalock-Taussig shunt in the Norwood procedure for hypoplastic left heart syndrome - influence on early and late haemodynamic status. Eur J Cardiothorac Surg. 2003; 23(5): 728-733, indexed in Pubmed: 12754025.

5. Pizarro C, Mroczek T, Malec E, et al. Right ventricle to pulmonary artery conduit reduces interim mortality after stage 1 Norwood for hypoplastic left heart syndrome. Ann Thorac Surg. 2004; 78(6): 1959-1963, doi: 10.1016/j.athoracsur.2004.06.020, indexed in Pubmed: 15561008

6. Tweddell JS, Sleeper LA, Ohye RG, et al. Pediatric Heart Network Investigators. Intermediate-term mortality and cardiac transplantation in infants with single-ventricle lesions: risk factors and their interaction with shunt type. J Thorac Cardiovasc Surg. 2012; 144(1): 152-159, doi: 10.1016/j.jtcvs.2012.01.016, indexed in Pubmed: 22341427.

7. Kolcz J, Skalski J. [Contemporary strategies of the hypoplastic left heart syndrome treatment]. Kardiol Pol. 2011; 69(3): 275-280. 
8. Malec E, Schmidt C, Lehner A, et al. Results of the Fontan operation with no early mortality in 248 consecutive patients. Kardiol Pol. 2017; 75(3): 255-260, doi: 10.5603/KP.a2016.0170, indexed in Pubmed: 27958617.

9. Mroczek T, Małota Z, Wójcik E, et al. Norwood with right ventricle-to-pulmonary artery conduit is more effective than Norwood with Blalock-Taussig shunt for hypoplastic left heart syndrome: mathematic modeling of hemodynamics. Eur J Cardiothorac Surg. 2011; 40(6): 1412-1417, doi: 10.1016/j.ejcts.2011.03.033, indexed in Pubmed: 21546259.

10. Ohye RG, Sleeper LA, Mahony L, et al. Pediatric Heart Network Investigators. Comparison of shunt types in the Norwood procedure for single-ventricle lesions. NEngl J Med. 2010; 362(21): 1980-1992, doi: 10.1056/NEJMoa0912461, indexed in Pubmed: 20505177.

11. Münsterer A, Kasnar-Samprec J, Hörer J, et al. Treatment of right ventricle to pulmonary artery conduit stenosis in infants with hypoplastic left heart syndrome. Eur J Cardiothorac Surg. 2013; 44(3): 468-471, doi: 10.1093/ejcts/ezt104, indexed in Pubmed: 23471153.

12. Schreiber C, Kasnar-Samprec J, Hörer J, et al. Ring-enforced right ventricle-to-pulmonary artery conduit in Norwood stage I reduces proximal conduit stenosis. Ann Thorac Surg. 2009; 88(5): 1541-1545, doi: 10.1016/j.athoracsur.2009.07.081, indexed in Pubmed: 19853109.

13. Tweddell J, Mitchell M, Woods R, et al. Construction of the Right Ventricle-to-Pulmonary Artery Conduit in the Norwood: The "Dunk" Technique. Operative Tech Thorac Cardiovasc Surg. 2012; 17(2): 81-98, doi: 10.1053/j.optechstcvs.2012.05.003.

14. Hasaniya NW, Shattuck H, Razzouk A, et al. Modification of ventricular-to-pulmonary shunt to minimize proximal conduit obstruction after stage I Norwood reconstruction. Ann Thorac
Surg. 2010; 89(1): e4-e6, doi: 10.1016/j.athoracsur.2009.08.079, indexed in Pubmed: 20103195.

15. Newburger JW, Sleeper LA, Frommelt PC, et al. Transplantation-free survival and interventions at 3 years in the single ventricle reconstruction trial. Circulation. 2014; 129(20): 2013-2020, doi: 10.1161/CIRCULATIONAHA.113.006191, indexed in Pubmed: 24705119

16. Baird CW, Myers PO, Borisuk M, et al. Ring-reinforced Sano conduit at Norwood stage I reduces proximal conduit obstruction. Ann Thorac Surg. 2015; 99(1): 171-179, doi: 10.1016/j. athoracsur.2014.08.078, indexed in Pubmed: 25441064.

17. Mascio CE, Spray TL. Distal dunk for right ventricle to pulmonary artery shunt in stage 1 palliation. Ann Thorac Surg. 2015; 100(6): 2381-2382, doi: 10.1016/j.athoracsur.2015.05.024, indexed in Pubmed: 26652546.

18. Ghanayem NS, Allen KR, Tabbutt S, et al. Interstage mortality after the Norwood procedure: Results of the multicenter Single Ventricle Reconstruction trial. J Thorac Cardiovasc Surg. 2012; 144(4): 896-906, doi: 10.1016/j.jtcvs.2012.05.020, indexed in Pubmed: 22795436.

19. Wilder TJ, McCrindle BW, Phillips AB, et al. Survival and right ventricular performance for matched children after stage-1 Norwood: Modified Blalock-Taussig shunt versus right-ventricle-to-pulmonary-artery conduit. J Thorac Cardiovasc Surg. 2015; 150(6): 1440-50, 1452.e1, doi: 10.1016/j.jtcvs.2015.06.069, indexed in Pubmed: 26254760.

20. Ravishankar C, Gerstenberger E, Sleeper LA, et al. Factors affecting Fontan length of stay: Results from the Single Ventricle Reconstruction trial. J Thorac Cardiovasc Surg. 2016; 151(3): 669-675.e1, doi: 10.1016/j.jtcvs.2015.09.061, indexed in Pubmed: 26519244

Cite this article as: Mroczek T, Sacharczuk J, Żurek R, et al. The “double dunk” technique for a right ventricle to pulmonary artery conduit implantation during the Norwood procedure reduces unintended shunt-related events. Kardiol Pol. 2018; 76(12): 1697-1704, doi: 10.5603/KP.a2018.0172.

\section{WHAT IS NEW?}

The use of a right ventricle to pulmonary artery conduit as a source of pulmonary blood flow, instead of the Blalock-Taussig shunt, during the Norwood procedure for the hypoplastic left heart syndrome revealed lower mortality rate, but higher incidence of unintended shunt-related interventions. We describe how refinements in the surgical technique may significantly reduce the incidence of unintended surgical and catheter-based, shunt-related reinterventions in patients with hypoplastic left heart syndrome after the Norwood procedure. We present the so-called "double dunk" technique of the right ventricle to pulmonary artery conduit implantation during the Norwood procedure, developed independently of other centres. We also suggest potential mechanisms leading to hypoplasia of pulmonary arteries after the Norwood procedure. The new surgical strategy presented herein allows better development of pulmonary arteries, which is considered to be an independent predictor of outcomes after the Fontan procedure. 